\title{
Neutrino oscillation tomography of the Earth with KM3NeT-ORCA
}

\section{S. Bourret ${ }^{a}$, João A. B. Coelho ${ }^{a}$, V. Van Elewyck ${ }^{*, a, b}$ for the KM3NeT Collaboration}

${ }^{a}$ APC, Université Paris Diderot, CNRS/IN2P3, CEA/Irfu, Observatoire de Paris, Sorbonne Paris Cité, France

${ }^{b}$ Institut Universitaire de France, 75005 Paris, France

E-mail: bourreteapc.in2p3.fr, jcoelhodapc.in2p3.fr,

elewyckeapc.univ-paris7.fr

KM3NeT-ORCA is a water-Cherenkov neutrino detector designed for studying the oscillation of atmospheric neutrinos, with the primary objective of measuring the neutrino mass ordering. Atmospheric neutrinos crossing the Earth undergo matter effects, modifying the pattern of their flavour oscillations. The study of the angular and energy distribution of neutrino events in ORCA can therefore provide tomographic information on the Earth's interior with an independent technique complementary to the standard geophysics methods.

This contribution presents an updated study of the potential of ORCA for Earth tomography, based on a full Monte Carlo simulation of the detector reponse and including systematic effects related to oscillation parameters, neutrino flux, cross-sections and detector performance, as well as possible degeneracies in the density measurement of different layers. Results show that after ten years of operation the proposed ORCA detector can measure the electron density with a precision of 5 to $6 \%$ in the mantle and 6 to $9 \%$ in the outer core - depending on the mass ordering.

35th International Cosmic Ray Conference - ICRC2017

10-20 July, 2017

Bexco, Busan, Korea

* Speaker. 


\section{Introduction}

ORCA (Oscillation Research with Cosmics in the Abyss) is the low-energy branch of KM3NeT, the next-generation neutrino Cherenkov detector currently under construction in the Mediterranean Sea. While the high-energy branch, ARCA (Astroparticle Research with Cosmics in the Abyss), will focus on the search for high-energy cosmic neutrino sources, ORCA's main goal will be the determination of the neutrino mass ordering $[1,2]$. The design of the ORCA detector considered in this work consists of a dense configuration of 115 vertical strings, with an horizontal spacing of $20 \mathrm{~m}$. The key detection element is the Digital Optical Module (DOM), a glass sphere comprising 31 photomultipliers (PMT) oriented towards all directions. Each string supports 18 (DOMs) with a vertical spacing of $9 \mathrm{~m}$, instrumenting a total mass of 5.7 Mton of seawater. With this configuration, ORCA will focus on the study of atmospheric neutrino oscillations in the energy range $\sim 1-100 \mathrm{GeV}$.

Due to coherent forward scattering on electrons, the flavour oscillations of atmospheric neutrinos propagating through the Earth matter are modified with respect to vacuum oscillations $[3,4,5$, 6]. An accurate measurement of this effect, based on the angular, energy and flavour distribution of neutrinos interactions detected with ORCA, can therefore provide sensitivity to the electron density $n_{e}$ in the different layers of matter traversed. This method is complementary to usual geophysical methods such as inversion of seismic data and geodetic measurements, which are used to infer the radial mass density profile. The ratio of electron density $n_{e}$ to mass density $\rho_{m}$ scales with the average proton to nucleon ratio (hereafter denoted $Z / A$ ), which depends on the chemical and isotopic composition of the medium:

$$
n_{e}=\frac{N_{A}}{m_{n}} \times \frac{Z}{A} \times \rho_{m}
$$

where $N_{A}$ is the Avogadro number and $m_{n}$ the nucleon mass.

Combining both measurements may thus lead to constraints on the compositional models of the inner Earth [7, 8]. In this work we use a reference geophysical model of the radial mass density profile in the Earth and we estimate the sensitivity of ORCA to the $Z / A$ ratio in the mantle and outer core.

\section{Simulation}

The event topologies of neutrino interactions with nuclei in seawater in the energy range of interest for ORCA can be separated in two broad classes. If the final state includes a sufficiently energetic (anti)muon, a track-like signature will be produced. This is the case for charged-current (CC) interactions of $v_{\mu} / \bar{v}_{\mu}$, and $v_{\tau} / \bar{v}_{\tau}$ with subsequent muonic decay of the (anti)tau lepton. Cascade-like events correspond to all other interaction channels, where only hadronic and electromagnetic showers are produced. This includes charged-current interactions of $v_{e} / \bar{v}_{e}$ and $v_{\tau} / \bar{v}_{\tau}$ with non-muonic decays, as well as neutral-current (NC) interactions. Dedicated event reconstruction algorithms have been developed for both track-like and cascade-like signatures. Event classification, together with rejection of down-going atmospheric muons, are based on a machine learning technique (Random Decision Forest). We refer the reader to [1] for details regarding simulations, detector resolutions and classification performance. 
The analysis presented here uses a full Monte Carlo simulation of the detector, that includes neutrino-nuclei interactions, propagation and decays of secondary particles, Cherenkov light generation and propagation, photo-detection and electronics effects. Dedicated software have been developed, based on widely used packages such as GENIE [9] and GEANT. They take into account extensively studied water properties at the detector site as well as DOM and PMT characteristics. In addition, optical background due to radioactive decays of ${ }^{40} \mathrm{~K}$ naturally present in seawater is simulated by adding uncorrelated (single-PMT) and correlated (inter-PMTs) random noise hits. Triggering is based on local space-time coincidence between PMT hits.

The analysis relies on the computation of energy $E$ and zenith angle $\theta_{z}$ (see Fig. 1) distributions of track-like and cascade-like events. The first step of this computation is the model of incoming atmospheric neutrino flux, which is taken from the HKKM2014 simulations ${ }^{1}$ [10]. The probabilities of neutrino flavour transitions are computed using a custom software framework, $O s c P r o b^{2}$. For a given zenith angle of incidence, the trajectory of a neutrino through the Earth is modelled along the corresponding baseline through a sequence of steps of constant electron density.

The model electron density inside the Earth used to infer the neutrino baselines is a radial model of the Earth with 42 concentric shells of constant electron density, where mass density values are fixed and follow the Preliminary Reference Earth Model (PREM) [11]. Additionnally, three chemical layers are defined, where the composition, and hence the $Z / A$ factor, are assumed to be uniform:

(a) solid iron inner core: $R=0-1221 \mathrm{~km}$

(b) liquid iron outer core: $R=1221-3480 \mathrm{~km}$

(c) silicate mantle (and crust): $R=3480-6368 \mathrm{~km}$

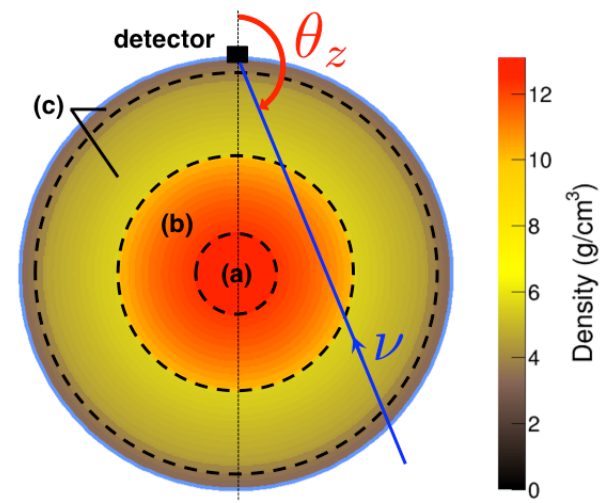

Figure 1: Density layers of the PREM.

The quantum evolution equations for the neutrino states are solved numerically for each step. Finally, the rate of events interacting around the detector is computed using neutrino-nucleon crosssections weighted for water molecules, obtained with the GENIE Monte Carlo generator [9].

The detector response is modelled using a full Monte Carlo approach. For each discrete channel (specified by the neutrino polarity $v / \bar{v}$, flavour, and event classification) a dedicated binned 4-dimensional response matrix is used for the transformation $\left(E_{\text {true }}, \theta_{\text {true }}\right) \rightarrow\left(E_{\text {reco }}, \theta_{\text {reco }}\right)$. These matrices are built using Monte Carlo generated events and the outcome of their processing through the reconstruction and classification algorithms, so that the ensemble of matrices account for detection and reconstruction efficiencies, misidentification probabilities and errors on reconstructed variables (including all correlations).

\footnotetext{
${ }^{1}$ Gran Sasso site without mountain, azimuth-averaged, solar minimum tables.

${ }^{2}$ See https://github.com/joaoabcoelho/OscProb
} 


\section{Analysis method}

The studies conducted so far aim at assessing ORCA's sensitivity to the measurement of uniform variations of $Z / A$ on large scales, i.e. inside a whole chemical layer. While the initial model for the 42 shells of constant matter density is kept as a basis, we introduce a rescaling of the electron density via the $Z / A$ factor, uniform over each chemical layer. In addition, several sources of systematic uncertainties are taken into account under the form of nuisance parameters, as listed in Table 1. Since no sizeable departure from solid iron is expected from geological models for the inner core, we do not consider its measurement but rather include it as a fixed parameter. We are thus left with two $Z / A$ parameters to be measured simultaneously: the mantle one ( $Z / A=0.496$ for the reference chemical composition, known as pyrolite) and the one of the outer core $(Z / A=0.466$ for the reference pure iron composition). In the fitting process of a given layer, the incomplete knowledge of the other layer Z/A is also considered as a source of systematic uncertainty.

Figure 2 shows the impact of varying by $5 \%$ the $Z / A$ in the outer core only (resp. mantle only) in terms of the expected signal, without including detector effects. At this stage, and for both layers, the dominant contribution to the signal comes from the $v_{\mu}+\bar{v}_{\mu}$ channel. However, the limited effective mass and energy/angular resolutions of ORCA at energies below $4 \mathrm{GeV}$ tend to wash out the signal in that region, resulting in comparable contributions of the track and cascade channels after the reconstruction step, as can be appreciated from Fig. 3 for the mantle.
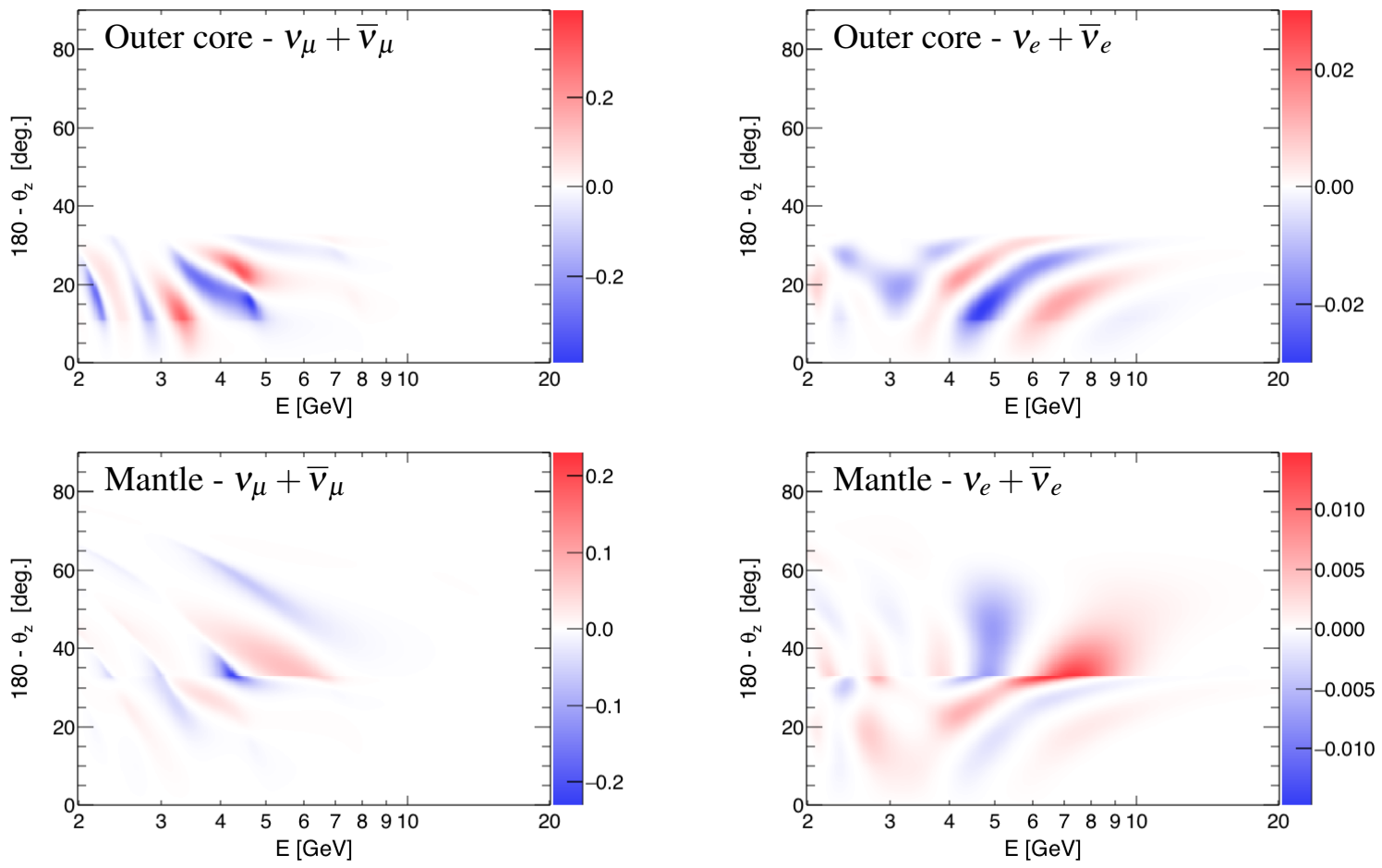

Figure 2: Expected signal, under the form of a signed $\chi^{2}($ bin $i)=\left(n_{A}^{i}-n_{B}^{i}\right) \times\left|n_{A}^{i}-n_{B}^{i}\right| / n_{A}^{i}$, obtained from the predicted number of interacting events (without including detector effects). Top plots: outer core, Bottom plots: mantle. Left plots: $v_{\mu}+\bar{v}_{\mu}$ channel, Right plots: $v_{e}+\bar{v}_{e}$ channel (mind the different scales on left and right plots). 

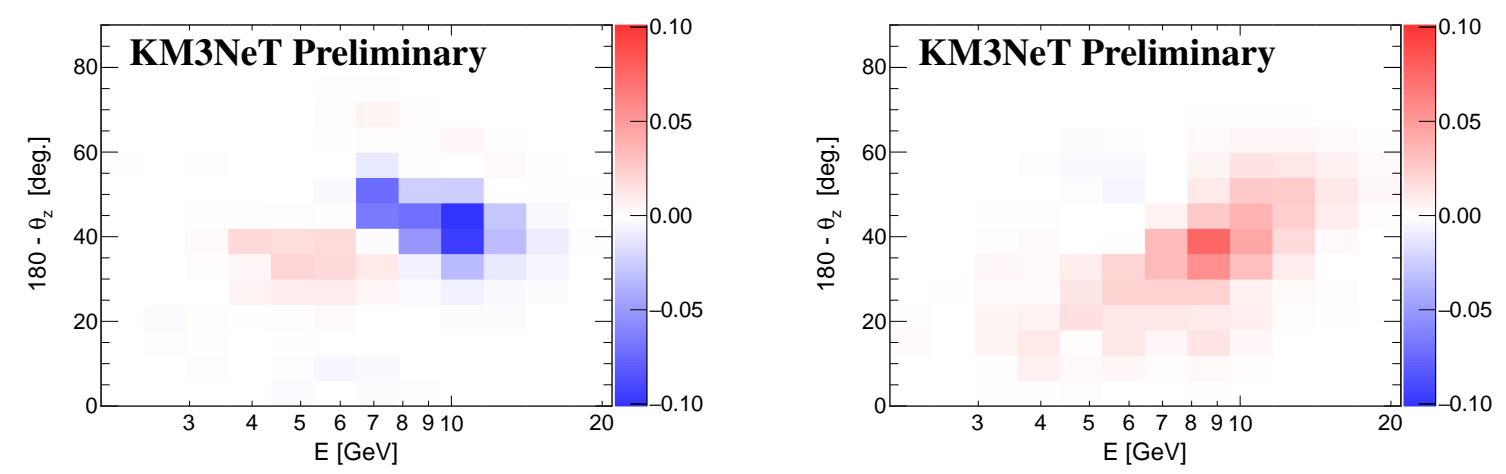

Figure 3: Expected signal, under the form of a signed $\chi^{2}($ bin $i)=\left(n_{A}^{i}-n_{B}^{i}\right) \times\left|n_{A}^{i}-n_{B}^{i}\right| / n_{A}^{i}$, obtained after reconstruction and classification of events into track (left) and cascade (right) channels, for 10 years of operation of the ORCA detector. Both channels contribute equally to the total sensitivity.

To compute the final sensitivity of ORCA to outer core and mantle composition, we use a binned $^{3} \log$-likelihood ratio method where the $\Delta \chi^{2}$ test statistic is profiled over the ensemble of nuisance parameters. Let us denote by $\lambda$ the measured parameter (either mantle or outer core $Z / A$ ) and $\left(\eta_{i}\right)$ the set of nuisance parameters - including the Z/A parameter in the other layer. Then for a given pseudo-data set $X$ and a given model hypothesis $\lambda=\lambda_{\text {hyp }}$ we use:

$$
\Delta \chi^{2}\left(X \mid \lambda_{\text {hyp }}\right)=-2 \ln \left[\frac{\max _{\eta_{i}} L\left(X \mid \lambda_{\text {hyp }}, \eta_{i}\right)}{\max _{\eta_{i}, \lambda} L\left(X \mid \lambda, \eta_{i}\right)}\right]=\min _{\eta_{i}} \chi^{2}\left(X \mid \lambda_{\text {hyp }}, \eta_{i}\right)-\min _{\eta_{i}, \lambda} \chi^{2}\left(X \mid \lambda, \eta_{i}\right) .
$$

The likelihood $L\left(X \mid \lambda, \eta_{i}\right)$ includes a statistical and a systematic part, i.e. we write:

$$
\chi^{2}\left(X \mid \lambda_{\text {hyp }}\right)=\chi_{\text {stat. }}^{2}+\chi_{\text {syst. }}^{2}
$$

where the statistical part combines both signal channels assuming bin-by-bin Poisson statistics:

$$
\chi_{\text {stat. }}^{2}=\sum_{\substack{\text { Tracks, } \\ \text { Cascades }}} \sum_{\substack{\text { bins } \log E \\ \text { bins } \theta_{z}}} 2\left[n_{\text {hyp }}-n_{X}+n_{X} \cdot \ln \left(\frac{n_{X}}{n_{\text {hyp }}}\right)\right],
$$

and the systematic part adds penalty terms to account for external constraints on some of the oscillation and systematics parameters, under the form of gaussian "prior" likelihood terms:

$$
\chi_{\text {syst. }}^{2}\left(\eta_{i}\right)=\sum_{i, \text { prior }} \frac{\left(\eta_{i}-\overline{\eta_{i}}\right)^{2}}{\sigma_{i}^{2}}
$$

where $\overline{\eta_{i}}$ and $\sigma_{i}$ are the mean and standard deviation of the prior for the parameter $\eta_{i}$. In this work we have chosen to evaluate the sensitivity using the average experiment (Asimov dataset) for pseudo-data $X$ instead of studying a large sample of randomized pseudo-experiments.

\footnotetext{
${ }^{3} \mathrm{We}$ choose to use 15 bins in $\theta_{z}$ in the range $\left[90^{\circ}-180^{\circ}\right]$ and 20 bins in $\log (E)$ in the range $[2-100] \mathrm{GeV}$, which is compatible with the detector resolutions in the energy range of interest.
} 


\begin{tabular}{lllll} 
parameter & treatment & true value & prior & $1 \sigma$ width \\
\hline $\operatorname{sign}\left(\Delta m_{31}^{2}\right)$ & fix & $\mathrm{NH}$ or $\mathrm{IH}$ & - & - \\
$\left|\Delta m_{31}^{2}\right|\left(\mathrm{eV}^{2}\right)$ & fitted & $2.4710^{-3}$ & no & - \\
$\Delta m_{21}^{2}\left(\mathrm{eV}^{2}\right)$ & fix & $7.5410^{-5}$ & - & - \\
$\theta_{13}\left(^{\circ}\right)$ & fitted & 8.51 & yes & 0.15 \\
$\theta_{12}\left(^{\circ}\right)$ & fix & 33.7 & - & - \\
$\theta_{23}\left(^{\circ}\right)$ & fitted & 41.4 & no & - \\
$\delta_{\mathrm{CP}}\left(^{\circ}\right)$ & fitted & 250 & no & - \\
Inner core $Z / A$ & fix & 0.466 & - & - \\
Overall normalization & fitted & 1 & yes & 0.10 \\
NC events normalization & fitted & 1 & yes & 0.05 \\
$v / \bar{v}$ polarity skew & fitted & 0 & yes & 0.03 \\
$e / \mu$ flavour skew & fitted & 0 & yes & 0.05 \\
\hline
\end{tabular}

Table 1: Treatment of oscillation and systematic parameters in this analysis. Most settings have been chosen in consistency with the existing analyses for the determination of the neutrino mass ordering and measurement of neutrino oscillation parameters with ORCA [2].

A summary of the treatment of oscillation and systematics parameters is given in Table 1. Since ORCA will be capable of determining the neutrino mass ordering with a confidence level of more than $3 \sigma$ in 3-4 years [2], and given the fact that the Earth tomography measurements need a significantly longer data-taking duration, it is safe to assume that the mass ordering is known and hence keep it fixed in the analysis.

\section{Results}
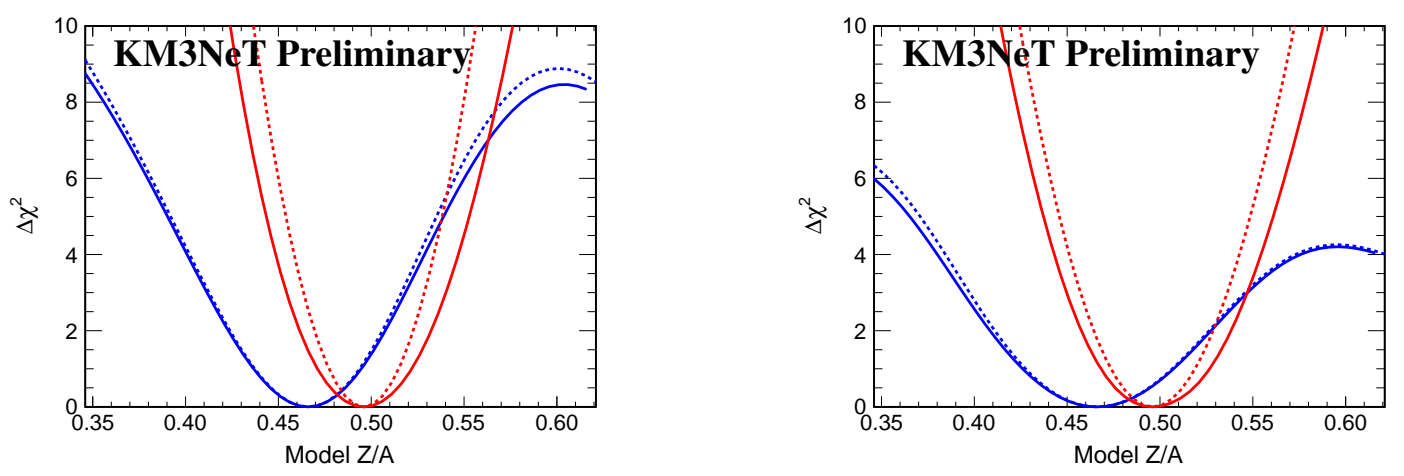

Figure 4: $\Delta \chi^{2}$ profiles for mantle (red) and outer core (blue), including only statistical effects (dotted lines) and statistical + systematic effects (solid lines), for 10 years of operation of the proposed ORCA detector. Left: normal ordering assumed, Right: inverted ordering assumed.

Figure 4 shows the $\Delta \chi^{2}$ profiles for the outer core and mantle measurements within the region of interest for the $Z / A$ parameter, with and without including the systematic uncertainties and assuming either normal or inverted neutrino mass ordering. A combination of these results is provided in Figure 5 (left panel). It is clear that ORCA will have better sensitivity to the electron 
density in the mantle than in the outer core, even considering that the measurement in the mantle is more affected by the systematic effects of Table 1 .
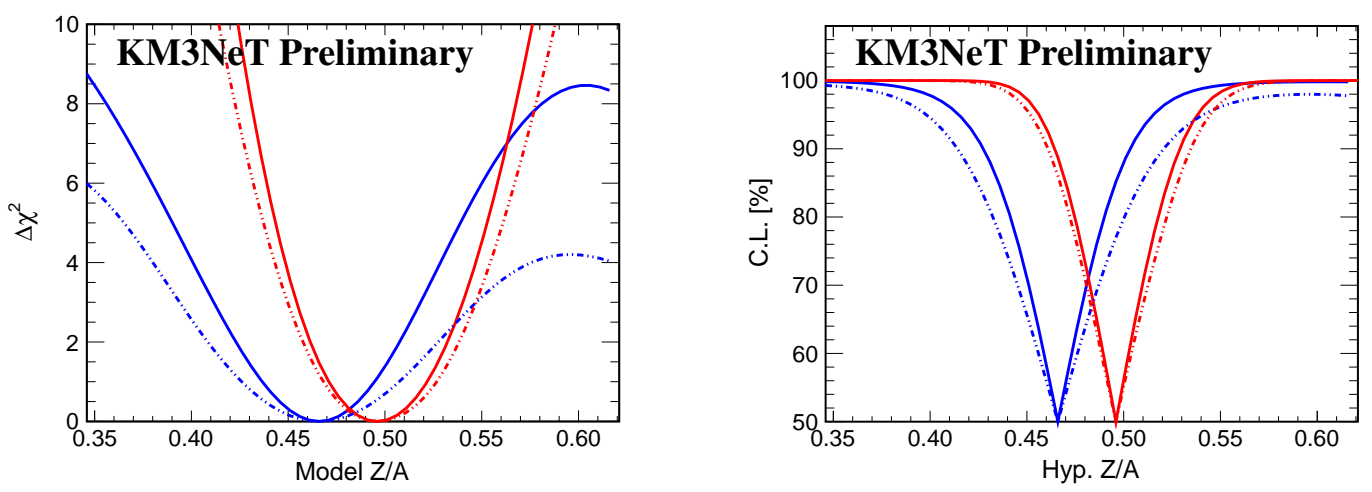

Figure 5: Left: $\Delta \chi^{2}$ profiles for mantle (red) and outer core (blue), for 10 years of operation of the ORCA detector. Right: Confidence level for rejecting the pyrolitic hypothesis for the mantle (red) and the pure iron hypothesis for the outer core (blue), after 10 years of operation of the ORCA detector. Both figures include systematic uncertainties. Solid lines are for normal ordering and dash-dotted lines for inverted ordering.

Figures 4 and 5 show that ORCA will have moderate sensitivity to the electron density in the outer core. Although the oscillations are enhanced for core-crossing neutrinos, the resonance energy $(\sim 2-4 \mathrm{GeV})$ is close to the detection threshold, which limits the statistics. Moreover, fast oscillations cannot be well resolved in this region due to limited angular and energy resolutions. The impact of systematic uncertainties is also shown to be negligible for the geophysically motivated range of values of $Z / A$, close to pure iron $(Z / A=0.466)$. High-density values for the outer core cannot be excluded with strong significance, especially in the case of inverted ordering, due to self-similarities of the oscillation probabilities as the electron density increases [8].

The composition of the mantle is usually approximated by the pyrolite rock model $(Z / A=$ 0.496). We report the estimated confidence level for rejecting this hypothesis as a function of the alternative true $Z / A$ in Fig. 5 (right panel). A confidence interval for the measurement of the $Z / A$ factor for both layers can be derived from the 1-dimensional $\Delta \chi^{2}$ profiles. After 10 years of operation, we estimate that the proposed ORCA detector will be able to measure the electron density in the Earth mantle to an accuracy of $\pm 5.1 \%$ ( $\pm 5.9 \%$ ) at $1 \sigma$ confidence level, assuming normal (inverted) mass ordering. In the outer core, an accuracy of $\pm 6.2 \%( \pm 8.8 \%)$ is achieved. As shown in Fig. 3, both track-like and cascade-like channels contribute to the signal.

These results are subject to further improvement once the updated performances of the ORCA detector are implemented. These will include e.g. new triggering algorithms that increase the detection efficiency of low-energy $(<5 \mathrm{GeV})$ events, particularly relevant for the present studies, and improved reconstruction methods.

\section{Acknowledgements}

The authors gratefully acknowledge financial support from the IdEx and LabEx UnivEarthS programs at Sorbonne Paris Cité (ANR-11-IDEX-0005-02, ANR-10-LABX-0023). 


\section{References}

[1] KM3NET collaboration, S. Adrian-Martinez et al., Letter of intent for KM3NeT 2.0, Journal of Physics G: Nuclear and Particle Physics 43 (2016) 084001, [1601.07459].

[2] KM3NET collaboration, A. Kouchner, Measuring the Neutrino Mass Ordering and other oscillation parameters with KM3NeT-ORCA, PoS(ICRC 2017) 1027.

[3] S. P. Mikheev and A. Yu. Smirnov, Resonance Amplification of Oscillations in Matter and Spectroscopy of Solar Neutrinos, Sov. J. Nucl. Phys. 42 (1985) 913-917.

[4] L. Wolfenstein, Neutrino oscillations in matter, Phys. Rev. D 17 (May, 1978) 2369-2374.

[5] S. T. Petcov, Diffractive - like (or parametric resonance - like?) enhancement of the Earth (day night) effect for solar neutrinos crossing the Earth core, Phys. Lett. B434 (1998) 321-332, [hep-ph/9805262].

[6] M. V. Chizhov and S. T. Petcov, Enhancing mechanisms of neutrino transitions in a medium of nonperiodic constant density layers and in the Earth, Phys. Rev. D63 (2001) 073003, [hep-ph/9903424].

[7] C. Rott, A. Taketa and D. Bose, Spectrometry of the Earth using Neutrino Oscillations, Scientific Reports 5 (Oct., 2015) 15225, [1502.04930].

[8] W. Winter, Atmospheric Neutrino Oscillations for Earth Tomography, Nucl. Phys. $\mathbf{B 9 0 8}$ (2016) 250-267.

[9] C. Andreopoulos et al., The GENIE Neutrino Monte Carlo Generator, Nucl. Instrum. Meth. A614 (2010) 87-104.

[10] M. Honda, M. Sajjad Athar, T. Kajita, K. Kasahara and S. Midorikawa, Atmospheric neutrino flux calculation using the NRLMSISE-00 atmospheric model, Phys. Rev. D92 (2015) 023004.

[11] A. M. Dziewonski and D. L. Anderson, Preliminary Reference Earth Model, Physics of the Earth and Planetary Interiors 25 (1981) 297-356. 